\title{
Molecular Characterization of Undifferentiated-Type Gastric Carcinoma
}

\author{
Gen Tamura, Kiyoshi Sato, Sunao Akiyama, Takashi Tsuchiya, Yasushi Endoh, \\ Osamu Usuba, Wataru Kimura, Satoshi Nishizuka, and Teiichi Motoyama
}

\author{
Departments of Pathology (GT, KS, SA, TT, YE, TM) and Surgery (KS, OU, WK), Yamagata University School of \\ Medicine, Yamagata, Japan; and Department of Microbiology and Molecular Genetics (SN), College of Medicine, \\ University of California, Irvine, California
}

\begin{abstract}
SUMMARY: As the great majority of gastric cancers develop histologically differentiated, and a significant proportion of differentiatedtype carcinomas progress to become undifferentiated, both histological types are likely to share several common genetic abnormalities, such as p53 mutations at advanced stages. However, a subset of gastric cancers develop as undifferentiated carcinomas, including signet-ring cell carcinoma and poorly differentiated adenocarcinoma, and the molecular pathogenesis of this tumor type remains largely unknown. To characterize the molecular features of undifferentiated-type gastric carcinomas that developed as undifferentiated-type, we examined for $p 53, A P C$, and epithelial (E)-cadherin gene mutations, microsatellite alterations including loss of heterozygosity ( $\mathrm{LOH}$ ) and microsatellite instability (MSI), and hypermethylation of the E-cadherin gene promoter in 26 early undifferentiated gastric carcinomas, consisting of 14 signet-ring cell carcinomas and 12 poorly differentiated adenocarcinomas. E-cadherin expression was evaluated immunohistochemically. p53 mutations were detected in only one poorly differentiated adenocarcinoma sample $(3.8 \% ; 1 / 26)$, whereas no APC or E-cadherin mutations were found. LOH was present only at D8S261 on the short arm of chromosome 8 in 2 of 14 (14\%) informative tumors, both of which were poorly differentiated adenocarcinomas, and MSI was not observed in any of the tumors. No signet-ring cell carcinomas have been found to carry gene mutations or microsatellite alterations. In contrast, hypermethylation of the E-cadherin promoter occurred in 69\% (18/26) of the tumors; $57 \%$ (8/14) of signet-ring cell carcinomas, and $83 \%(10 / 12)$ of poorly differentiated adenocarcinomas, and was significantly associated with loss or reduced expression of E-cadherin. Thus, whereas tumor suppressor gene mutation, LOH, and MSI were less common in undifferentiated-type early gastric carcinomas, epigenetic inactivation of $E$-cadherin via promoter hypermethylation may be an early critical event in the development of undifferentiated tumors. (Lab Invest 2001, 81:593-598).
\end{abstract}

G astric cancers are classified histopathologically as either differentiated carcinomas forming tubular or papillary structures or undifferentiated carcinomas in which such structures are inconspicuous (Nakamura et al, 1968). Different genetic pathways are thought to be involved in the development of these histological types because they exhibit different frequencies of genetic alterations, including mutation of the K-ras oncogene and $A P C$ tumor suppressor gene, amplification of $c$-erbB- 2 and c-met oncogenes, deletion of chromosomal arms, and microsatellite instability (MSI) (Tahara, 1995). However, significant discrepancies in the frequency of genetic alterations among differentiated and undifferentiated carcinomas have been reported (Han et al, 1993; Horii et al, 1992; Kihana et al, 1991; Nakatsuru et al, 1992; Victor et al, 1990; Yamamoto et al, 1999). A significant proportion of the differentiated carcinomas progressed to be-

Received January 22, 2001.

This study was supported by a Grant-in-Aid for Cancer Research (No. 10-3) from the Ministry of Health and Welfare and by a Grant-in-Aid (No. 12670154) from the Ministry of Education, Science, Sports and Culture of Japan.

Address reprint requests to: Dr. Gen Tamura, Department of Pathology, Yamagata University School of Medicine, Iida-nishi 2-2-2, Yamagata 990-9585, Japan.E-mail: gtamura@med.id.yamagata-u.ac.jp come undifferentiated, so that the proportion of differentiated carcinomas decreased with increasing tumor size through the increased prevalence of undifferentiated tumors (Endoh et al, 2000b). Thus, advancedstage undifferentiated gastric carcinomas originate from both differentiated and undifferentiated tumor types, and both histological types at advanced stages share common genetic alterations such as p53 mutation, which occurs as an early event in differentiated carcinomas of the stomach (Tamura et al, 1995). However, it is clear that a subset of gastric cancers develop primarily as undifferentiated tumors, and early undifferentiated carcinomas rarely exhibit p53 mutations despite high frequencies in early and advanced differentiated-type and advanced undifferentiatedtype carcinomas (Maesawa et al, 1995; Uchino et al, 1993).

Recently it was reported that aberrant DNA methylation of promoter region $\mathrm{CpG}$ islands may serve as an alternate mechanism to coding region mutation for the inactivation of tumor suppressor or tumor-related genes (Graff et al, 1997; Herman et al, 1996, 1998; Stirzaker et al, 1997). Also, we have previously reported that transcriptional silencing of the E-cadherin via promoter hypermethylation frequently occurred in undifferentiated-type carcinomas of the stomach (Tamura et al, 2000). Because a subset of colorectal 
and gastric cancers exhibited the CpG island methylator phenotype (Toyota et al, 1999a, 1999b), it is possible that undifferentiated-type gastric carcinomas were involved.

To characterize the molecular features of undifferentiated gastric carcinomas that developed as undifferentiated carcinomas, we examined for mutations in p53, APC, and E-cadherin genes, for microsatellite alterations (loss of heterozygosity [LOH] and MSI), and for hypermethylation of the $E$-cadherin gene promoter in 26 early gastric undifferentiated carcinomas.

\section{Results}

\section{Mutations of p53, APC, and E-cadherin}

Polymerase chain reaction single-strand conformation polymorphism (PCR-SSCP) detected p53 mutation in only one poorly differentiated adenocarcinoma (3.8\%; 1/26) (Fig. 1A). DNA sequencing of the shifted band revealed a silent mutation at codon 249 (CGG to $A G G$ ) in exon 7 (Fig. 1B). No APC or E-cadherin mutations were found.

\section{Microsatellite Alterations}

Frequencies of informative cases at each microsatellite locus were $54 \%$ (14/26 informative cases) at

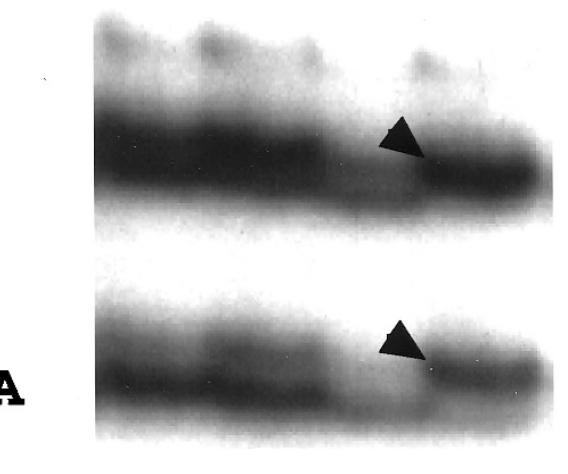

\section{A}

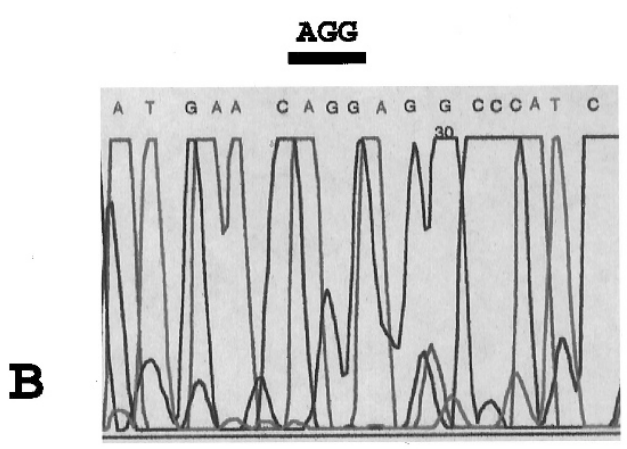

Figure 1.

Polymerase chain reaction single-strand conformation polymorphism (PCRSSCP) (A) and sequencing (B) analyses of exon 7 of the $p 53$ gene. Sequencing of the mobility shift band (arrowheads) revealed a silent mutation at codon 249 (CGG to $A G G$ ) in exon7.
D4S404, 92\% (24/26) at D5S409, 77\% (22/26) at MYH7, 54\% (14/26) at D8S261, 23\% (6/26) at D9S171, 46\% (12/26) at D16S397, 62\% (16/26) at TP53, and 69\% (18/26) at D18S46. LOH was present only at D8S261 on the short arm of chromosome 8 in 2 of $14(14 \%)$ informative tumors, both of which were poorly differentiated adenocarcinomas, and no MSI was present in any of the tumors (Fig. 2).

\section{Promoter Hypermethylation and Expression of E-cadherin}

Hypermethylation of the E-cadherin promoter occurred in $69 \%(18 / 26)$ of tumors; $57 \%(8 / 14)$ of signetring cell carcinomas, and $83 \%(10 / 12)$ of poorly differentiated adenocarcinomas (Fig. 3). Direct sequencing confirmed the retention of cytosines at CpGs in methylated PCR products and the conversion of all the cytosines to thymines in unmethylated PCR products from several gastric carcinoma samples similarly to our previous findings (Tamura et al, 2000) (data not shown). Loss or reduced E-cadherin expression was observed immunohistochemically in 10 of 12 tumors $(83 \%)$ with promoter hypermethylation (Fig. 4) and none of 3 tumors without hypermethylation $(p=0.022$, by Fisher's exact test).

\section{Discussion}

Differentiated- and undifferentiated-type gastric carcinomas show different epidemiological characteristics and may be the result of different genetic pathways. Differentiated-type carcinomas are thought to arise from gastric epithelial cells that have undergone intestinal metaplasia and may be associated with Helicobacter pylori infection (Correa, 1995), whereas undifferentiatedtype carcinomas may arise from native gastric epithelial cells. Because advanced undifferentiated-type carcinomas are composed of cells originating from both differentiated and undifferentiated tumors, it is necessary to study purely undifferentiated-type carcinomas to clarify their molecular pathogenesis. In addition, pure undifferentiated-type carcinomas often show scirrhous
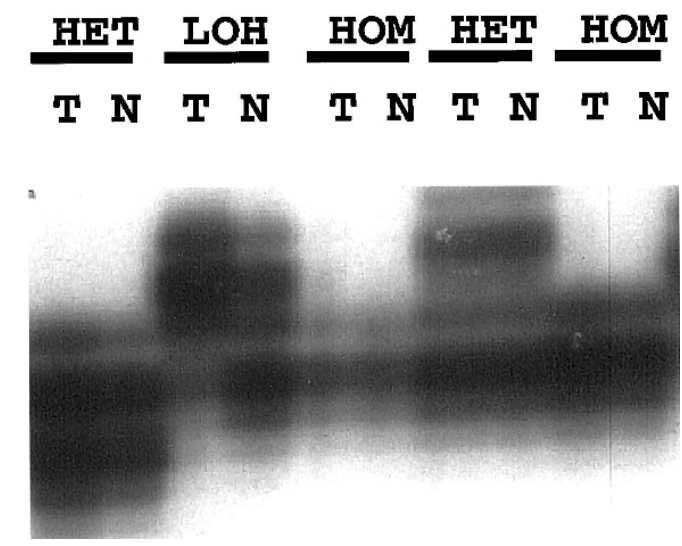

Figure 2.

Examples of microsatellite analysis at D8S261. Analysis of tumor (T) and normal (N) DNA from five patients is shown. HET, heterozygosity; LOH, loss of heterozygosity; HOM, homozygosity. 


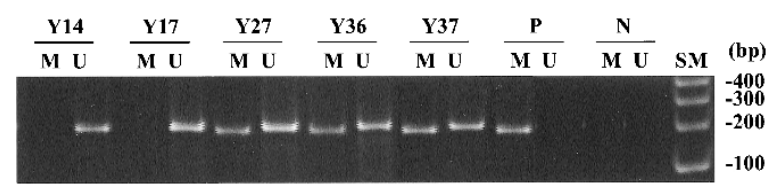

Figure 3.

Methylation-specific polymerase chain reaction (MSP) in five undifferentiated carcinomas. Methylated alleles were present in Y27, Y36, and Y37. M, methylated PCR products; U, unmethylated PCR products; P, positive control (Sss I-treated DNA); N, negative control (unmodified DNA).

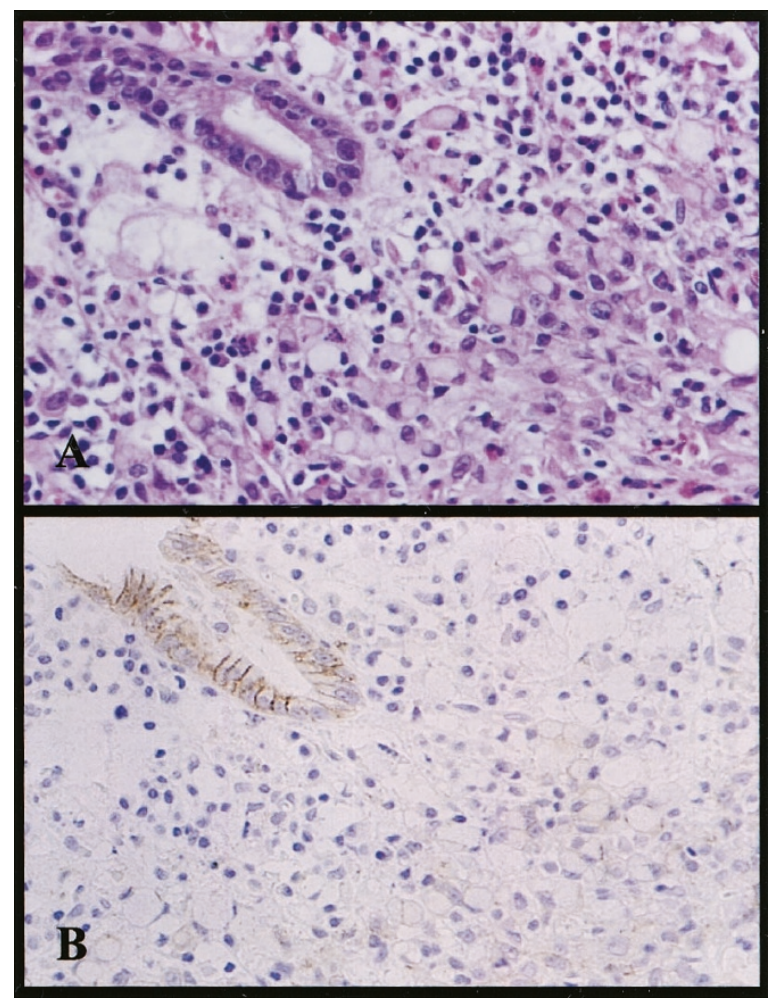

Figure 4.

Histological feature (A) and immunohistochemical reactivity for E-cadherin (B) in a case of intramucosal signet ring cell carcinoma. Whereas foveolar epithelial cells show strong membranous staining, signet ring carcinoma cells show marked reduction in E-cadherin expression.

growth that makes it difficult to investigate genetic alterations because of low tumor cellularity at advanced stages. Therefore, we attempted to investigate genetic and epigenetic alterations in early-stage undifferentiated-type carcinomas with relatively high tumor cellularity.

Mutation of $p 53$ is one of the most prevalent genetic alterations in human gastric carcinomas, and is considered to be early critical event in the development of differentiated-type gastric carcinomas (Tamura et al, 1995). The frequency of p53 mutations in early and advanced differentiated-type gastric carcinomas is consistent at around $40 \%$ of tumors, similar to that in advanced undifferentiated-type carcinomas (Maesawa et al, 1995; Uchino et al, 1993). Thus, the paucity of $p 53$ mutations is characteristic of early-stage undifferentiated carcinomas. In the present study, we have confirmed the rarity of $p 53$ mutations as we found only one in the 12 poorly differentiated adenocarcinomas tested $(8.3 \%)$, and that was a silent mutation and therefore probably did not contribute to carcinogenesis, similar to a previous report (Maesawa et al, 1995). Mutation of $A P C$ is an early critical event in both familial and sporadic forms of colorectal tumorigenesis via the adenoma-carcinoma sequence (Miyoshi et al, 1992a, 1992b). Although APC mutations are rare in extracolonic cancers, mutated forms have been found in 3 of 31 undifferentiated-type carcinomas (9.7\%), including 10 signet-ring cell carcinomas (Horii et al, 1992), and in 1 of 25 undifferentiated-type carcinomas (4.0\%), including 15 signet-ring cell carcinomas (Maesawa et al, 1995). We detected no APC mutations in our undifferentiated-type early gastric carcinomas.

Deletions have been frequently reported for several chromosomal loci, including $5 \mathrm{q}, 7 \mathrm{q}, 17 \mathrm{p}, 18 \mathrm{q}$, and $21 q$, in differentiated-type carcinomas of the stomach (Nishizuka et al, 1998; Sakata et al, 1997; Tamura et al, 1996a). However, few reports have focused on LOH in undifferentiated-type carcinomas, probably because of the difficulty of performing $\mathrm{LOH}$ analysis on tissue samples with low tumor cellularity. Nonetheless, frequent $\mathrm{LOH}$ on $5 \mathrm{q}$ has been reported for this tumor type at advanced stages with or without tumor cell enrichment (McKie et al, 1993; Tamura et al, 1993). In our study, LOH was detected only on $8 p$ in 2 of 16 (12.5\%) informative tumors, both of which were poorly differentiated adenocarcinomas, and no other incidences of LOH were found at any other loci, including $5 \mathrm{q}$. The frequency of $\mathrm{MSI}$ in gastric cancers varies among reports, so that reports have suggested that undifferentiated-type carcinomas exhibit more frequent MSI than differentiated (Han et al, 1993), or vice versa (Yamamoto et al, 1999). In contrast to our previous report of microsatellite analysis in which LOH was present in $48 \%(29 / 61)$ and high frequency MSI (MSI-H) in $23 \%(14 / 61)$ of early differentiated-type gastric carcinomas (Ohmura et al, 2000), we detected few LOH and no MSI in 26 early undifferentiated-type carcinomas in the present study. We have also reported that the cellular phenotypes of differentiatedtype gastric carcinomas were dependent on genetic pathway, so that differentiated foveolar-type carcinomas followed the mutator pathway characterized by MSI (Endoh et al, 2000a; Ohmura et al, 2000). Because differentiated foveolar- and undifferentiated-type carcinomas, both of which may possibly arise from foveolar epithelial cells, rarely exhibit LOH, these carcinomas may constitute the CpG island methylator phenotype (Toyota et al, 1999a, 1999b). Thus, promoter hypermethylation of $h M L H 1$ in gastric foveolar epithelial cells may lead to differentiated foveolar-type carcinomas, whereas promoter hypermethylation of E-cadherin may lead to undifferentiated-type carcinomas.

The E-cadherin gene is frequently found to be inactivated via the classic two-hit mechanism in undifferentiated-scattered (diffuse)-type gastric carcinomas at advanced stages, but not in differentiated or undifferentiated-adherent-type gastric carcinomas (Becker et al, 1994; Tamura et al, 1996b). E-cadherin mutations were infrequent in undifferentiated gastric 
carcinomas including signet-ring cell carcinomas at their early stages (Tamura et al 1996b; Muta et al, 1996), and E-cadherin mutations were not detected in our present study. Because E-cadherin mutations were detected in the undifferentiated component, but not in the differentiated component, of mixed differentiated/undifferentiated carcinomas, it is possible that E-cadherin is mutated when differentiated carcinomas progress to become undifferentiated (Machado et al, 1999). In addition, frequent inactivation of E-cadherin via hypermethylation of promoter region $\mathrm{CpG}$ islands, which acts as an alternate mechanism to coding region mutations, has recently been reported for undifferentiated-scatteredtype gastric carcinomas (Tamura et al, 2000). E-cadherin promoter hypermethylation occurs frequently in undifferentiated-type carcinomas, at similar rates in early and advanced stages (Tamura et al, 2000), and can also occur in noncancerous gastric epithelia (Suzuki et al, 1999). In the present study, E-cadherin promoter hypermethylation was detected in $69 \%(18 / 26)$ of tumors, $57 \%(8 / 14)$ of signet-ring cell carcinomas, and $83 \%$ $(10 / 12)$ of poorly differentiated adenocarcinomas. We have previously reported that hypermethylation of this region correlated well with loss or reduction of E-cadherin protein expression by Western blotting (Tamura et al, 2000), and we confirmed this association with reduced E-cadherin expression by immunohistochemistry in the present study. Loss or reduction of E-cadherin expression is known to occur frequently in undifferentiated-type gastric carcinomas, even at early stages (Blok et al, 1999). Therefore, epigenetic inactivation of E-cadherin via promoter hypermethylation may play a major role in the development of purely undifferentiated-type carcinomas of the stomach, and mutations of this gene may be involved in the progression of differentiated to undifferentiated carcinomas.

In conclusion, mutations in tumor suppressor genes, $\mathrm{LOH}$, and $\mathrm{MSI}$ are less common in early undifferentiated-type gastric carcinomas, and epigenetic inactivation of $E$-cadherin via promoter hyper- methylation may be an early critical event in the development of this tumor type.

\section{Materials and Methods}

\section{Samples and DNA Extraction}

Twenty-six gastric carcinomas and corresponding noncancerous tissues were obtained surgically from 26 patients. Parts of these tissues were stored at $-80^{\circ} \mathrm{C}$ for DNA extraction, and the remaining tissues were fixed in $10 \%$ buffered formalin for histological examination. The carcinomas studied were histologically undifferentiated carcinomas, including 14 signetring cell carcinomas and 12 poorly differentiated adenocarcinomas, and consisted of 14 intramucosal and 12 submucosal invasive carcinomas. All these tumor samples were histologically confirmed to contain more than 50\% tumor cells, and samples with low tumor cellularity (less than 50\%) were excluded from the present study. DNA was isolated by standard proteinase $\mathrm{K}$ digestion and phenol/chloroform extraction procedures.

\section{PCR-SSCP Analysis of p53, APC, and E-Cadherin}

p53, $A P C$, and E-cadherin gene mutations were studied by PCR-SSCP analysis according to established protocols (Tamura et al, 1995, 1996a, 1996b). Briefly, extracted DNA (100 ng) was amplified in $10 \mu \mathrm{l} 1 \times$ PCR Gold Buffer (Perkin-Elmer Cetus, Norwalk, Connecticut) containing $10 \mathrm{pm}$ each primer, $1 \mathrm{~mm} \mathrm{MgCl}_{2}, 0.2$ $\mathrm{mm}$ each deoxynucleotide triphosphate, $0.5 \mu \mathrm{l}\left[\alpha^{-32} \mathrm{P}\right]-$ dCTP, and 0.5 units AmpliTaq Gold (Perkin-Elmer Cetus). Primer sequences are listed in Table 1. PCR was performed for 35 cycles in a GeneAmp PCR System 2400 thermocycler (Perkin-Elmer Cetus) consisting of denaturation at $95^{\circ} \mathrm{C}$ for 15 seconds, annealing at $55^{\circ} \mathrm{C}$ for 15 seconds, and extension at $72^{\circ} \mathrm{C}$ for 30 seconds, after initial heating at $95^{\circ} \mathrm{C}$ for 10 minutes to activate the AmpliTaq Gold. PCR prod-

Table 1. Oligonucleotide Primers $\left(5^{\prime}-3^{\prime}\right)$ for PCR-SSCP

\begin{tabular}{cll}
\hline & \multicolumn{1}{c}{ Upstream } & \multicolumn{1}{c}{ Downstream } \\
\hline$p 53$ & & \\
Exon 5 & TTCCTCTTCCTGCAGTACTCC & GCCCCAGCTGCTCACCATCG \\
Exon 6 & CACTGATTGCTCTTAGGT & AGTTGCAAACCAGACCTCA \\
Exon 7 & TCCTAGGTTGGCTCTGAC & CAGTGGCTCCTGACCTGGA \\
Exon 8 & CCTATCCTGAGTAGTGGTAA & \\
APC & AGACTTATTGTGTAGCTCGACT \\
Exon $15^{a}$ & TCTGTCAGTTCACTTGATAGAC & ATGGTTCACTCTGAACGGA \\
Exon $15^{b}$ & CATTTGATTCTTTAGGCTGC \\
E-cadherin & & \\
Exon 6 & TCCTCATCGAGCTCAAGTC & GGGTCCAAAGAACCTAAGAG \\
Exon 7 & TGCCCAGTCCCAAAGTGCAG & TCCACACCCTCTGGATCCTC \\
Exon 8 & CGGTGGCTAGTGTTCCTGG & GGTTGGAACACCTCTAA \\
Exon 9 & GACACATCTCTTTGCTCTGC & \\
\hline
\end{tabular}

PCR-SSCP, polymerase chain reaction-single strand conformation polymorphism.

a Codon 1260-1410.

${ }^{b}$ Codon 1389-1547. 
ucts were diluted 10 -fold with gel-loading buffer, heated to $94^{\circ} \mathrm{C}$ for 2 minutes, and stored on ice until analysis. Electrophoresis was performed on $6 \%$ neutral polyacrylamide gels at $60 \mathrm{~W}$ for 2 to 4 hours. Gels were dried and exposed to x-ray film at $-80^{\circ} \mathrm{C}$ overnight. Second-round PCR was performed to confirm the reproducibility.

\section{DNA Sequencing Analysis}

Mobility-shift SSCP bands were excised from gels and subjected to a second round of PCR amplification using the same primers as used above, as described (Tamura et al, 1995). The reamplified PCR products were purified and sequenced using a terminator cycle sequencing kit (dRhodamine Terminator Cycle Sequencing FS Ready Reaction Kits, Perkin-Elmer Cetus) and an automated DNA sequencer (310 Genetic Analyzer, Perkin-Elmer Cetus).

\section{Microsatellite Analysis}

Eight microsatellite markers, D4S404, D5S409, MYH7, D8S261, D9S171, D16S397, TP53, and D18S46 were investigated. Primers were obtained from MapPairs (Research Genetics, Huntsville, Alabama). PCR was performed by the same procedure as described for PCR-SSCP. Electrophoresis was performed on $6 \%$ polyacrylamide gels containing $7 \mathrm{M}$ urea at $60 \mathrm{~W}$ for 2 to 2.5 hours. $\mathrm{LOH}$ and $\mathrm{MSI}$ were defined as visible changes as previously described (Tamura et al, 1995).

\section{Methylation-Specific Polymerase Chain Reaction (MSP)}

DNA methylation patterns in the E-cadherin promoter region were determined by MSP (Tamura et al, 2000). MSP distinguishes unmethylated from methylated alleles of a given gene based on sequence alterations produced by bisulfite treatment of DNA, which converts unmethylated, but not methylated, cytosines to uracil. Subsequent PCR then uses primers specific to either methylated or unmethylated DNA sequences (Herman et al, 1996). Primer sequences used were 5'GGTGAATITTAGTTAATTAGCGGTAC-3' and 5'CATAACTAACCGAAAACGCCG-3' for methylated DNA, and 5'-GGTAGGTGAATITTAGTTAATTAGTGGTA-3' and 5'-ACCCATAACTAACCAAAAACACCA-3' for unmethylated DNA. The PCR-amplified region for methylated (204 bp) and unmethylated (211 bp) alleles contained $19 \mathrm{CpG}$ dinucleotides, including $5 \mathrm{CpGs}$ within the primer annealing sites. Primer sets spanned the transcriptional start site and were designed to include methylation sites that best corresponded with transcriptional silencing of the E-cadherin gene (Baylin et al, 1998). Aliquots of genomic DNA (2 $\mu \mathrm{g})$ were denatured by treatment with $\mathrm{NaOH}$ and modified by sodium bisulfite. DNA samples were then purified using Wizard DNA purification resin (Promega, Madison, Wisconsin), again treated with $\mathrm{NaOH}$, precipitated with ethanol, and resuspended in water. Extracted DNA (100 ng) were amplified under the same conditions as described for PCR-SSCP. PCR products were directly loaded onto nondenaturating $6 \%$ polyacrylamide gels, stained with ethidium bro- mide, and visualized under UV illumination. Peripheral blood DNA $(100 \mu \mathrm{g})$ was treated by Sss I Methylase (New England BioLabs, Beverly, Massachusetts) according to the manufacturer's protocol. Sss I-treated DNA was modified by bisulfite as described above and used as a positive control for methylated DNA. Unmodified DNA was used as a negative control for MSP.

\section{Immunohistochemistry}

Immunohistochemistry was preformed on formalinfixed, paraffin-embedded tissue sections using the standard labeled streptavidin-biotin system (Nichirei, Tokyo, Japan) on 15 of the 26 early undifferentiated gastric carcinomas. Mouse monoclonal antibody to E-cadherin (clone HECD-1; Takara, Kyoto, Japan) was used at 1:250 dilution after antigen retrieval by microwave. Expression was evaluated according to previously published methods (Oka et al, 1992).

\section{References}

Baylin SB, Herman JG, Graff JR, Vertino PM, and Issa A (1998). Alterations in DNA methylation: a fundamental aspect of neoplasia. Adv Cancer Res 72:141-196.

Becker KF, Atkinson MJ, Reich U, Becker I, Nekarda H, Siewert JR, and Hofler H (1994). E-cadherin gene mutations provide clues to diffuse type gastric carcinomas. Cancer Res 54:3845-3852.

Blok P, Craanen ME, Dekker W, and Tytgat GN (1999). Loss of $\mathrm{E}$-cadherin expression in early gastric cancer. Histopathology $34: 410-415$.

Correa P (1995). Helicobacter pylori and gastric carcinogenesis. Am J Surg Pathol 19(Suppl 1):37-43.

Endoh Y, Sakata K, Tamura G, Ohmura K, Ajioka Y, Watanabe H, and Motoyama T (2000a). Cellular phenotypes of differentiated-type adenocarcinomas and precancerous lesions of the stomach are dependent on the genetic pathways. J Pathol 191:257-263.

Endoh Y, Tamura G, Watanabe H, and Motoyama T (2000b). Author's reply. J Pathol 191:467-468.

Graff JR, Herman JG, Myohanen S, Baylin SB, and Vertino PM (1997). Mapping patterns of CpG island methylation in normal and neoplastic cells implicates both upstream and downstream regions in de novo methylation. $\mathrm{J}$ Biol Chem 272:22322-22329.

Han HJ, Yanagisawa A, Kato Y, Park JG, and Nakamura Y (1993). Genetic instability in pancreatic cancer and poorly differentiated type of gastric cancer. Cancer Res 53:50875089.

Herman JG, Graff JR, Myohanen S, Kelkin BD, and Baylin SB (1996). Methylation-specific PCR: A novel PCR assay for methylation status of $\mathrm{CpG}$ islands. Proc Natl Acad Sci USA 93:9821-9826.

Herman JG, Umar A, Polyak K, Graff JR, Ahuja, N, Issa J-PJ, Markowitz S, Willson JKV, Hamilton SR, Kinzler KW, Kane M F, Kolodner RD, Vogelstein B, Kunkel TA, and Baylin SB (1998). Incidence and functional consequence of $h M L H 1$ promoter hypermethylation in colorectal carcinoma. Proc Natl Acad Sci USA 95:6870-6875. 
Horii A, Nakatsuru S, Miyoshi Y, Ichii S, Nagase H, Kato $\mathrm{Y}$, Yanagisawa A, and Nakamura $Y$ (1992). The APC gene, responsible for familial adenomatous polyposis, is mutated in human gastric cancer. Cancer Res 52:3231-3233.

Kihana T, Tsuda H, Hirota T, Shimosato Y, Sakamoto H, Terada M, and Hirohashi S (1991). Point mutation of c-Ki-ras oncogene in gastric adenoma and adenocarcinoma with tubular differentiation. Jpn J Cancer 82:308-314.

Machado JC, Soares P, Carneiro F, Rocha A, Beck S, Blin N, Berx G, and Sobrinho-Simoes M (1999). E-cadherin gene mutations provide a genetic basis for the phenotypic divergence of mixed gastric carcinomas. Lab Invest 79:459-465.

Maesawa C, Tamura G, Suzuki Y, Ogasawara S, Sakata K, Kashiwaba M, and Satodate R (1995). The sequential accumulation of genetic alterations characteristic of the colorectal adenoma-carcinoma sequence does not occur between gastric adenoma and adenocarcinoma. J Pathol 176:249-258.

McKie AB, Filipe MI, and Lemoine NR (1993). Abnormalities affecting the $A P C$ and $M C C$ tumour suppressor gene loci on chromosome $5 \mathrm{q}$ occur frequently in gastric cancer but not in pancreatic cancer. Int J Cancer 55:598-603.

Miyoshi Y, Ando H, Nagase H, Nishisho I, Horii A, Miki Y, Mori T, Utsunomiya J, Baba S, Petersen G, Hamilton SR, Kinzler KW, Vogelstein B, and Nakamura Y (1992a). Germ-line mutations of the $A P C$ in 53 familial adenomatous polyposis patients. Proc Natl Acad Sci USA 89:4452-4456.

Miyoshi Y, Nagase H, Ando H, Horii A, Ichii S, Nakatsuru S, Aoki T, Miki Y, Mori T, and Nakamura Y (1992b). Somatic mutations of the APC gene in colorectal tunors: Mutation cluster region in the APC gene. Hum Mol Genet 1:229-233.

Muta $H$, Noguchi M, Kanai $Y$, Ochiai A, Nawata $H$, and Hirohashi S (1996). E-cadherin gene mutation in signet ring cell carcinoma of the stomach. Jpn J Cancer Res 87:843848.

Nakamura K, Sugano H, and Takagi K (1968). Carcinoma of the stomach in incipient phase: Its histogenesis and histological appearances. GANN 59:251-258.

Nakatsuru S, Yanagisawa A, Ichii S, Tahara E, Kato Y, Nakamura $Y$, and Horii A (1992). Somatic mutation of the $A P C$ gene in gastric cancer: Frequent mutations in very well differentiated adenocarcinoma and signet-ring cell carcinoma. Hum Mol Genet 1:559-563.

Nishizuka S, Tamura G, Terashima M, and Satodate R (1998). Loss of heterozygosity during the development and progression of differentiated adenocarcinoma of the stomach. J Pathol 185:38-43.

Ohmura K, Tamura G, Endoh Y, Sakata K, Takahashi T, and Motoyama T (2000). Microsatellite alterations in differentiated-type adenocarcinomas and precancerous lesions of the stomach: With special reference to cellular phenotype. Hum Pathol 31:1031-1035.

Oka H, Shiozaki H, Kobayashi K, Tahara H, Tamura S, Miyata M, Doki Y, lihara K, Matsuyoshi N, Hirano S, Takeichi M, and Mori T (1992). Immunohistochemical evaluation of E-cadherin adhesion molecule expression in human gastric cancer. Virchows Arch A Pathol Anat Histopathol 42:149-156.

Sakata K, Tamura G, Nishizuka S, Maesawa C, Suzuki Y, Iwaya T, Terashima M, Saito K, and Satodate R (1997). Commonly deleted regions on the long arm of chromosome 21 in differentiated adenocarcinoma of the stomach. Genes Chromosomes Cancer 18:318-321.
Stirzaker C, Millar DS, Paul CL, Warnecke PM, Harrison J, Vincent PC, Frommer M, and Clark SJ (1997). Extensive DNA methylation spanning the $R b$ promoter in retinoblastoma tumors. Cancer Res 57:2229-2237.

Suzuki H, Itoh F, Toyota M, Kikuchi T, Kakiuchi H, Hinoda Y, and Imai K (1999). Distinct methylation pattern and microsatellite instability in sporadic gastric cancer. Int $\mathrm{J}$ Cancer 83:309-313.

Tahara E (1995). Genetic alterations in human gastrointestinal cancers: The application to molecular diagnosis. Cancer 75S:1410-1417.

Tamura G, Maesawa C, Suzuki Y, Ogasawara S, Terashima M, Saito K, and Satodate R (1993). Primary gastric carcinoma cells frequently lose heterozygosity at the $A P C$ and $M C C$ genetic loci. Jpn J Cancer Res 84:1015-1018.

Tamura G, Ogasawara S, Nishizuka S, Sakata K, Maesawa C, Suzuki Y, Terashima M, Saito K, and Satodate R (1996a). Two distinct regions of deletion on the long arm of chromosome 5 in differentiated adenocarcinomas of the stomach. Cancer Res 56:612-615.

Tamura G, Sakata K, Maesawa C, Suzuki Y, Terashima M, Satoh K, Sekiyama S, Suzuki A, Eda Y, and Satodate R (1995). Microsatellite alterations in adenoma and differentiated adenocarcinoma of the stomach. Cancer Res 55:1933-1936.

Tamura G, Sakata K, Nishizuka S, Maesawa C, Suzuki Y, Iwaya T, Terashima M, Saito K, and Satodate R (1996b). Inactivation of the $E$-cadherin gene in primary gastric carcinomas and gastric carcinoma cell lines. Jpn J Cancer Res 87:1153-1159.

Tamura G, Yin J, Wang S, Fleisher AS, Zou T, Abraham JM, Kong D, Smolinski KN, Wilson KT, James SP, Silverberg SG, Nishizuka S, Terashima M, Motoyama T, and Meltzer SJ (2000). $E$-Cadherin gene promoter hypermethylation in primary human gastric carcinomas. J Natl Cancer Inst 92:569-753.

Toyota M, Ahuja N, Ohe-Toyota M, Herman JG, Baylin SB, and Issa JP (1999a). CpG island methylator phenotype in colorectal cancer. Proc Natl Acad Sci USA 96:8681-8686.

Toyota M, Ahuja N, Suzuki H, Itoh F, Ohe-Toyota M, Imai K, Baylin SB, and Issa JP (1999b). Aberrant methylation in gastric cancer associated with the $\mathrm{CpG}$ island methylator phenotype. Cancer Res 59:5438-5442.

Uchino S, Noguchi M, Ochiai A, Saito T, Kobayashi M, and Hirohashi S (1993). p53 mutation in gastric cancer: A genetic model for carcinogenesis is common to gastric and colorectal cancer. Int J Cancer 54:759-764.

Victor T, Du Toit R, Jordaan AM, Bester AJ, and van Helden PD (1990). No evidence for point mutations in codons 12, 13, and 61 of the ras gene in a high-incidence area for esophageal and gastric cancers. Cancer Res 15:4911-4914.

Yamamoto H, Perez-Piteira J, Yoshida T, Terada M, Itoh F, Imai K, and Perucho M (1999). Gastric cancers of the microsatellite mutator phenotype display characteristic genetic and clinical features. Gastroenterology 116:1348-1357. 